\title{
Coefficient estimates for families of bi-univalent functions defined by Ruscheweyh derivative operator
}

\author{
Serap Bulut, Abbas Kareem Wanas
}

\begin{abstract}
The main purpose of this manuscript is to find upper bounds for the second and third Taylor-Maclaurin coefficients for two families of holomorphic and bi-univalent functions associated with Ruscheweyh derivative operator. Further, we point out certain special cases for our results.
\end{abstract}

\section{INTRODUCTION}

Indicate by $\mathcal{A}$ the collection of all holomorphic functions in the open unit disk

$$
U=\{z \in \mathbb{C}:|z|<1\}
$$

that have the form

$$
f(z)=z+\sum_{n=2}^{\infty} a_{n} z^{n} .
$$

Further, assume that $\mathcal{S}$ stands for the sub-collection of the set $\mathcal{A}$ containing of functions in $U$ satisfying (1) which are univalent in $U$.

For a function $f \in \mathcal{A}$ defined by (1), the Ruscheweyh derivative operator $\mathcal{R}^{\delta}: \mathcal{A} \longrightarrow \mathcal{A}$ (see [14]) is defined as follows:

$$
\begin{aligned}
& \mathcal{R}^{\delta} f(z)=z+\sum_{n=2}^{\infty} \frac{\Gamma(\delta+n)}{(n-1) ! \Gamma(\delta+1)} a_{n} z^{n}, \\
& \left(\delta \in \mathbb{N}_{0}=\{0,1,2, \ldots\}:=\mathbb{N} \cup\{0\}, z \in U\right) .
\end{aligned}
$$

According to the Koebe One-Quarter Theorem (see [6]) every function $f \in \mathcal{S}$ has an inverse $f^{-1}$ defined by

$f^{-1}(f(z))=z \quad(z \in U) \quad$ and $\quad f\left(f^{-1}(w)\right)=w \quad\left(|w|<r_{0}(f), r_{0}(f) \geq \frac{1}{4}\right)$.

2020 Mathematics Subject Classification. Primary: 30C45; Secondary: 30C50.

Key words and phrases. Holomorphic function, bi-univalent function, coefficient estimates, Ruscheweyh derivative operator.

Full paper. Received 30 November 2020, revised 23 January 2021, accepted 4 February 2021, available online 16 March 2021. 
For the inverse function $f^{-1}$, we have

$$
\begin{aligned}
g(w)=f^{-1}(w)= & w-a_{2} w^{2}+\left(2 a_{2}^{2}-a_{3}\right) w^{3} \\
& -\left(5 a_{2}^{3}-5 a_{2} a_{3}+a_{4}\right) w^{4}+\cdots .
\end{aligned}
$$

For $f \in \mathcal{A}$, if both $f$ and $f^{-1}$ are univalent in $U$, we say that $f$ is bi-univalent function in $U$. We indicate by $\Sigma$ the family of bi-univalent functions in $U$ given by (1). In fact, Srivastava et al. [22] have actually revived the study of holomorphic and bi-univalent functions in recent years, it was followed by such works as those by Frasin and Aouf [8], Murugusundaramoorthy et al. [13], Srivastava and Wanas [25] and others (see, for example $[1,3,4,5,9,10,11,12,15,16,17,18,19,20,21,23,14,24,26$, $27,28,29,30,31,32,33])$. We notice that the family $\Sigma$ is not empty. Some examples of functions in the family $\Sigma$ are

$$
\frac{z}{1-z}, \quad \frac{1}{2} \log \left(\frac{1+z}{1-z}\right) \text { and }-\log (1-z),
$$

with the corresponding inverse functions

$$
\frac{w}{1+w}, \quad \frac{e^{2 w}-1}{e^{2 w}+1} \text { and } \frac{e^{w}-1}{e^{w}},
$$

respectively. Other common examples of functions is not a member of $\Sigma$ are

$$
z-\frac{z^{2}}{2} \text { and } \frac{z}{1-z^{2}}
$$

Until now, the coefficient estimate problem for each of the following Taylor-Maclaurin coefficients $\left|a_{n}\right|(n=3,4, \ldots)$ for functions $f \in \Sigma$ is still an open problem.

We require the following lemma that will be used to prove our main results.

Lemma 1 ([6]). If $h \in \mathcal{P}$, then $\left|c_{k}\right| \leq 2$ for each $k \in \mathbb{N}$, where $\mathcal{P}$ is the family of all functions $h$ holomorphic in $U$ for which

$$
\Re(h(z))>0 \quad(z \in U),
$$

where

$$
h(z)=1+c_{1} z+c_{2} z^{2}+\cdots \quad(z \in U) .
$$

2. Coefficient Estimates for the Functions Family

$$
\mathcal{Q}_{\Sigma}(\gamma, \mu, \lambda, \delta ; \alpha)
$$

Throughout this section, we suppose that

$$
\mu \geq 1, \gamma \geq 0,0<\alpha \leq 1, \delta \in \mathbb{N}_{0} \text { and } \lambda \in \mathbb{C} \backslash\{0\} .
$$

Definition 1. A function $f \in \Sigma$ given by (1) is called in the family $\mathcal{Q}_{\Sigma}(\gamma, \mu, \lambda, \delta ; \alpha)$ if it fulfills the conditions:

$$
\left|\arg \left(1+\frac{1}{\lambda}\left[(1-\mu) \frac{\mathcal{R}^{\delta} f(z)}{z}+\mu\left(\mathcal{R}^{\delta} f(z)\right)^{\prime}+\gamma z\left(\mathcal{R}^{\delta} f(z)\right)^{\prime \prime}-1\right]\right)\right|<\frac{\alpha \pi}{2}
$$


and

(4) $\left|\arg \left(1+\frac{1}{\lambda}\left[(1-\mu) \frac{\mathcal{R}^{\delta} g(w)}{w}+\mu\left(\mathcal{R}^{\delta} g(w)\right)^{\prime}+\gamma w\left(\mathcal{R}^{\delta} g(w)\right)^{\prime \prime}-1\right]\right)\right|<\frac{\alpha \pi}{2}$, where $z, w \in U$ and $g=f^{-1}$ is given by (2).

Remark 1. It should be remarked that the family $\mathcal{Q}_{\Sigma}(\gamma, \mu, \lambda, \delta ; \alpha)$ is a generalization of well-known families consider earlier. These families are:

(1) for $\delta=0$ and $\mu=\lambda=1$, the family $\mathcal{Q}_{\Sigma}(\gamma, \mu, \lambda, \delta ; \alpha)$ reduce to the family $\mathcal{H}_{\Sigma}(\alpha, \gamma)$ which was considered by Frasin [7];

(2) for $\gamma=\delta=0$ and $\lambda=1$, the family $\mathcal{Q}_{\Sigma}(\gamma, \mu, \lambda, \delta ; \alpha)$ reduce to the family $\mathcal{B}_{\Sigma}(\alpha, \mu)$ which was given by Frasin and Aouf [8];

(3) for $\gamma=\delta=0$ and $\mu=\lambda=1$, the family $\mathcal{Q}_{\Sigma}(\gamma, \mu, \lambda, \delta ; \alpha)$ reduce to the family $\mathcal{H}_{\Sigma}^{\alpha}$ which was investigated by Srivastava et al. [22].

Theorem 1. Let $f \in \mathcal{Q}_{\Sigma}(\gamma, \mu, \lambda, \delta ; \alpha)$ be given by $(1)$. Then

$$
\left|a_{2}\right| \leq \frac{2 \alpha|\lambda|}{\sqrt{\left|\alpha \lambda(\delta+2)(\delta+1)(1+2 \mu+6 \gamma)+(1-\alpha)(\delta+1)^{2}(1+\mu+2 \gamma)^{2}\right|}}
$$

and

$$
\left|a_{3}\right| \leq \frac{4 \alpha^{2}|\lambda|^{2}}{(\delta+1)^{2}(1+\mu+2 \gamma)^{2}}+\frac{4 \alpha|\lambda|}{(\delta+2)(\delta+1)(1+2 \mu+6 \gamma)} .
$$

Proof. In the light of the conditions (3) and (4), we deduce that

(5) $1+\frac{1}{\lambda}\left[(1-\mu) \frac{\mathcal{R}^{\delta} f(z)}{z}+\mu\left(\mathcal{R}^{\delta} f(z)\right)^{\prime}+\gamma z\left(\mathcal{R}^{\delta} f(z)\right)^{\prime \prime}-1\right]=[p(z)]^{\alpha}$

and

(6) $1+\frac{1}{\lambda}\left[(1-\mu) \frac{\mathcal{R}^{\delta} g(w)}{w}+\mu\left(\mathcal{R}^{\delta} g(w)\right)^{\prime}+\gamma w\left(\mathcal{R}^{\delta} g(w)\right)^{\prime \prime}-1\right]=[q(w)]^{\alpha}$,

where $g=f^{-1}$ is given by (2) and $p, q$ in $\mathcal{P}$ have the following series representations:

$$
p(z)=1+p_{1} z+p_{2} z^{2}+p_{3} z^{3}+\cdots
$$

and

$$
q(w)=1+q_{1} w+q_{2} w^{2}+q_{3} w^{3}+\cdots .
$$

Comparing the corresponding coefficients of (5) and (6) yields

$$
\begin{gathered}
\frac{(\delta+1)(1+\mu+2 \gamma)}{\lambda} a_{2}=\alpha p_{1}, \\
\frac{(\delta+2)(\delta+1)(1+2 \mu+6 \gamma)}{2 \lambda} a_{3}=\alpha p_{2}+\frac{\alpha(\alpha-1)}{2} p_{1}^{2},
\end{gathered}
$$




$$
-\frac{(\delta+1)(1+\mu+2 \gamma)}{\lambda} a_{2}=\alpha q_{1}
$$

and

$$
\frac{(\delta+2)(\delta+1)(1+2 \mu+6 \gamma)}{2 \lambda}\left(2 a_{2}^{2}-a_{3}\right)=\alpha q_{2}+\frac{\alpha(\alpha-1)}{2} q_{1}^{2} .
$$

In view of (9) and (11), we conclude that

$$
p_{1}=-q_{1}
$$

and

$$
\frac{2(\delta+1)^{2}(1+\mu+2 \gamma)^{2}}{\lambda^{2}} a_{2}^{2}=\alpha^{2}\left(p_{1}^{2}+q_{1}^{2}\right) .
$$

Also, by using (10) and (12), together with (14), we find that

$$
\begin{aligned}
& \frac{(\delta+2)(\delta+1)(1+2 \mu+6 \gamma)}{\lambda} a_{2}^{2}=\alpha\left(p_{2}+q_{2}\right)+\frac{\alpha(\alpha-1)}{2}\left(p_{1}^{2}+q_{1}^{2}\right) \\
& =\alpha\left(p_{2}+q_{2}\right)+\frac{(\alpha-1)(\delta+1)^{2}(1+\mu+2 \gamma)^{2}}{\alpha \lambda^{2}} a_{2}^{2} .
\end{aligned}
$$

Further computations show that

$$
a_{2}^{2}=\frac{\alpha^{2} \lambda^{2}\left(p_{2}+q_{2}\right)}{\alpha \lambda(\delta+2)(\delta+1)(1+2 \mu+6 \gamma)+(1-\alpha)(\delta+1)^{2}(1+\mu+2 \gamma)^{2}},
$$

By taking the absolute value of (15) and applying Lemma 1 for the coefficients $p_{2}$ and $q_{2}$, we have

$$
\left|a_{2}\right| \leq \frac{2 \alpha|\lambda|}{\sqrt{\left|\alpha \lambda(\delta+2)(\delta+1)(1+2 \mu+6 \gamma)+(1-\alpha)(\delta+1)^{2}(1+\mu+2 \gamma)^{2}\right|}} .
$$

To determinate the bound on $\left|a_{3}\right|$, by subtracting (12) from (10), we get

$$
\frac{(\delta+2)(\delta+1)(1+2 \mu+6 \gamma)}{\lambda}\left(a_{3}-a_{2}^{2}\right)=\alpha\left(p_{2}-q_{2}\right)+\frac{\alpha(\alpha-1)}{2}\left(p_{1}^{2}-q_{1}^{2}\right) .
$$

Now, substituting the value of $a_{2}^{2}$ from (14) into (16) and using (13), we deduce that

$$
a_{3}=\frac{\alpha^{2} \lambda^{2}\left(p_{1}^{2}+q_{1}^{2}\right)}{2(\delta+1)^{2}(1+\mu+2 \gamma)^{2}}+\frac{\alpha \lambda\left(p_{2}-q_{2}\right)}{(\delta+2)(\delta+1)(1+2 \mu+6 \gamma)} .
$$

Taking the absolute value of (17) and applying Lemma 1 once again for the coefficients $p_{1}, p_{2}, q_{1}$ and $q_{2}$, it follows that

$$
\left|a_{3}\right| \leq \frac{4 \alpha^{2}|\lambda|^{2}}{(\delta+1)^{2}(1+\mu+2 \gamma)^{2}}+\frac{4 \alpha|\lambda|}{(\delta+2)(\delta+1)(1+2 \mu+6 \gamma)} .
$$

Remark 2. In Theorem 1, if we choose

(1) $\delta=0$ and $\mu=\lambda=1$, then we have the results obtained by Frasin [7, Theorem 2.2]; 
(2) $\gamma=\delta=0$ and $\lambda=1$, then we have the results obtained by Frasin and Aouf [8, Theorem 2.2];

(3) $\gamma=\delta=0$ and $\mu=\lambda=1$, then we obtain the results obtained by Srivastava et al. [22, Theorem 1].

\section{Coefficient Estimates for the Functions Family}

$$
\mathcal{Q}_{\Sigma}^{*}(\gamma, \mu, \lambda, \delta ; \beta)
$$

Throughout this section, we suppose that

$$
\mu \geq 1, \gamma \geq 0,0 \leq \beta<1, \delta \in \mathbb{N}_{0} \text { and } \lambda \in \mathbb{C} \backslash\{0\}
$$

Definition 2. A function $f \in \Sigma$ given by (1) is called in the family $\mathcal{Q}_{\Sigma}^{*}(\gamma, \mu, \lambda, \delta ; \beta)$ if it fulfills the conditions

$$
\Re\left\{1+\frac{1}{\lambda}\left[(1-\mu) \frac{\mathcal{R}^{\delta} f(z)}{z}+\mu\left(\mathcal{R}^{\delta} f(z)\right)^{\prime}+\gamma z\left(\mathcal{R}^{\delta} f(z)\right)^{\prime \prime}-1\right]\right\}>\beta
$$

and

(19) $\Re\left\{1+\frac{1}{\lambda}\left[(1-\mu) \frac{\mathcal{R}^{\delta} g(w)}{w}+\mu\left(\mathcal{R}^{\delta} g(w)\right)^{\prime}+\gamma w\left(\mathcal{R}^{\delta} g(w)\right)^{\prime \prime}-1\right]\right\}>\beta$,

where $z, w \in U$ and $g=f^{-1}$ is given by (2).

Remark 3. It should be remarked that the family $\mathcal{Q}_{\Sigma}^{*}(\gamma, \mu, \lambda, \delta ; \beta)$ is a generalization of well-known families consider earlier. These families are:

(1) for $\delta=0$ and $\lambda=1$, the family $\mathcal{Q}_{\Sigma}^{*}(\gamma, \mu, \lambda, \delta ; \beta)$ reduce to the family $\mathcal{N}_{\Sigma}(\beta, \mu, \gamma)$ which was defined by Bulut [2];

(2) for $\delta=0$ and $\mu=\lambda=1$, the family $\mathcal{Q}_{\Sigma}^{*}(\gamma, \mu, \lambda, \delta ; \beta)$ reduce to the family $\mathcal{H}_{\Sigma}(\gamma, \beta)$ which was considered by Frasin [7];

(3) for $\gamma=\delta=0$ and $\lambda=1$, the family $\mathcal{Q}_{\Sigma}^{*}(\gamma, \mu, \lambda, \delta ; \beta)$ reduce to the family $\mathcal{B}_{\Sigma}(\beta, \mu)$ which was given by Frasin and Aouf [8];

(4) for $\gamma=\delta=0$ and $\mu=\lambda=1$, the family $\mathcal{Q}_{\Sigma}^{*}(\gamma, \mu, \lambda, \delta ; \beta)$ reduce to the family $\mathcal{H}_{\Sigma}(\beta)$ which was investigated by Srivastava et al. [22].

Theorem 2. Let $f \in \mathcal{Q}_{\Sigma}^{*}(\gamma, \mu, \lambda, \delta ; \beta)$ be given by $(1)$. Then

$$
\left|a_{2}\right| \leq\left\{\begin{array}{cl}
2 \sqrt{\frac{|\lambda|(1-\beta)}{(\delta+2)(\delta+1)(1+2 \mu+6 \gamma)},}, & 0 \leq \beta \leq 1-\frac{(\delta+1)(1+\mu+2 \gamma)^{2}}{|\lambda|(\delta+2)(1+2 \mu+6 \gamma)}, \\
\frac{2|\lambda|(1-\beta)}{(\delta+1)(1+\mu+2 \gamma)}, & 1-\frac{(\delta+1)(1+\mu+2 \gamma)^{2}}{|\lambda|(\delta+2)(1+2 \mu+6 \gamma)} \leq \beta<1
\end{array}\right.
$$

and

$$
\left|a_{3}\right| \leq \frac{4|\lambda|(1-\beta)}{(\delta+2)(\delta+1)(1+2 \mu+6 \gamma)}
$$


Proof. In the light of the conditions (18) and (19), there are $p, q \in \mathcal{P}$ such that

$$
\begin{aligned}
& 1+\frac{1}{\lambda}\left[(1-\mu) \frac{\mathcal{R}^{\delta} f(z)}{z}+\mu\left(\mathcal{R}^{\delta} f(z)\right)^{\prime}+\gamma z\left(\mathcal{R}^{\delta} f(z)\right)^{\prime \prime}-1\right] \\
& =\beta+(1-\beta) p(z)
\end{aligned}
$$

and

$$
\begin{aligned}
& 1+\frac{1}{\lambda}\left[(1-\mu) \frac{\mathcal{R}^{\delta} g(w)}{w}+\mu\left(\mathcal{R}^{\delta} g(w)\right)^{\prime}+\gamma w\left(\mathcal{R}^{\delta} g(w)\right)^{\prime \prime}-1\right] \\
& =\beta+(1-\beta) q(w),
\end{aligned}
$$

where $g=f^{-1}$ is given by (2), $p(z)$ and $q(w)$ have the forms (7) and (8), respectively. Comparing the corresponding coefficients in (20) and (21) yields

$$
\begin{gathered}
\frac{(\delta+1)(1+\mu+2 \gamma)}{\lambda} a_{2}=(1-\beta) p_{1}, \\
\frac{(\delta+2)(\delta+1)(1+2 \mu+6 \gamma)}{2 \lambda} a_{3}=(1-\beta) p_{2}, \\
-\frac{(\delta+1)(1+\mu+2 \gamma)}{\lambda} a_{2}=(1-\beta) q_{1}
\end{gathered}
$$

and

$$
\frac{(\delta+2)(\delta+1)(1+2 \mu+6 \gamma)}{2 \lambda}\left(2 a_{2}^{2}-a_{3}\right)=(1-\beta) q_{2} .
$$

From (22) and (24), we get

and

$$
p_{1}=-q_{1}
$$

Adding (23) and (25), we obtain

$$
\frac{2(\delta+1)^{2}(1+\mu+2 \gamma)^{2}}{\lambda^{2}} a_{2}^{2}=(1-\beta)^{2}\left(p_{1}^{2}+q_{1}^{2}\right) .
$$

Hence, we find from (26) and (27) that

$$
\frac{(\delta+2)(\delta+1)(1+2 \mu+6 \gamma)}{\lambda} a_{2}^{2}=(1-\beta)\left(p_{2}+q_{2}\right) .
$$

$$
a_{2}^{2}=\frac{\lambda^{2}(1-\beta)^{2}\left(p_{1}^{2}+q_{1}^{2}\right)}{2(\delta+1)^{2}(1+\mu+2 \gamma)^{2}}
$$

and

$$
a_{2}^{2}=\frac{\lambda(1-\beta)\left(p_{2}+q_{2}\right)}{(\delta+2)(\delta+1)(1+2 \mu+6 \gamma)},
$$

respectively. By applying Lemma 1 for the coefficients $p_{2}$ and $q_{2}$, we deduce that

$$
\left|a_{2}\right| \leq \frac{2|\lambda|(1-\beta)}{(\delta+1)(1+\mu+2 \gamma)}
$$


and

$$
\left|a_{2}\right| \leq 2 \sqrt{\frac{|\lambda|(1-\beta)}{(\delta+2)(\delta+1)(1+2 \mu+6 \gamma)}},
$$

respectively. To determinate the bound on $\left|a_{3}\right|$, by subtracting (25) from (23), we get

$$
\frac{(\delta+2)(\delta+1)(1+2 \mu+6 \gamma)}{\lambda}\left(a_{3}-a_{2}^{2}\right)=(1-\beta)\left(p_{2}-q_{2}\right),
$$

or equivalently

$$
a_{3}=a_{2}^{2}+\frac{\lambda(1-\beta)\left(p_{2}-q_{2}\right)}{(\delta+2)(\delta+1)(1+2 \mu+6 \gamma)} .
$$

Substituting the value of $a_{2}^{2}$ from (26) and (27) into (28), it follows that

$$
a_{3}=\frac{\lambda^{2}(1-\beta)^{2}\left(p_{1}^{2}+q_{1}^{2}\right)}{2(\delta+1)^{2}(1+\mu+2 \gamma)^{2}}+\frac{\lambda(1-\beta)\left(p_{2}-q_{2}\right)}{(\delta+2)(\delta+1)(1+2 \mu+6 \gamma)}
$$

and

$$
a_{3}=\frac{2 \lambda(1-\beta) p_{2}}{(\delta+2)(\delta+1)(1+2 \mu+6 \gamma)},
$$

respectively. By applying Lemma 1 once again for the coefficients $p_{1}, p_{2}, q_{1}$ and $q_{2}$, we deduce that

$$
\left|a_{3}\right| \leq \frac{4|\lambda|^{2}(1-\beta)^{2}}{(\delta+1)^{2}(1+\mu+2 \gamma)^{2}}+\frac{4|\lambda|(1-\beta)}{(\delta+2)(\delta+1)(1+2 \mu+6 \gamma)}
$$

and

$$
\left|a_{3}\right| \leq \frac{4|\lambda|(1-\beta)}{(\delta+2)(\delta+1)(1+2 \mu+6 \gamma)},
$$

respectively. This completes the proof.

Remark 4. In Theorem 2, if we choose

(1) $\delta=0$ and $\lambda=1$, then we have the results obtained by Bulut [2, Theorem 3.4];

(2) $\delta=0$ and $\mu=\lambda=1$, then we have improvements of the results obtained by Frasin [7, Theorem 3.2];

(3) $\gamma=\delta=0$ and $\lambda=1$, then we have improvements of the results obtained by Frasin and Aouf [8, Theorem 3.2];

(4) $\gamma=\delta=0$ and $\mu=\lambda=1$, then we have improvements of the results obtained by Srivastava et al. [22, Theorem 2]. 


\section{Conclusion}

In this investigation, we have introduced and defined two a certain families of holomorphic and bi-univalent functions in the open unit disk $U$ associated with Ruscheweyh derivative operator. We have then derived the initial coefficient estimations for functions belonging to these families. Further by specializing the parameters, several consequences of these families are mentioned.

\section{REFERENCES}

[1] E. A. Adegani, S. Bulut, A. A. Zireh, Coefficient estimates for a subclass of analytic bi-univalent functions, Bulletin of the Korean Mathematical Society, 55 (2) (2018), 405-413.

[2] S. Bulut, Faber polynomial coefficient estimates for a subclass of analytic bi-univalent functions, Filomat, 30 (6) (2016), 1567-1575.

[3] S. Bulut, Coefficient estimates for general subclasses of $m$-fold symmetric analytic bi-univalent functions, Turkish Journal of Mathematics, 40 (2016), 1386-1397.

[4] S. Bulut, A new general subclass of analytic bi-univalent functions, Turkish Journal of Mathematics, 43 (2019), 1330-1338.

[5] E. Deniz, Certain subclasses of bi-univalent functions satisfying subordinate conditions, Journal of Classical Analysis, 2 (2013), 49-60.

[6] P. L. Duren, Univalent Functions, Grundlehren der Mathematischen Wissenschaften, Band 259, Springer Verlag, New York, Berlin, Heidelberg and Tokyo, 1983.

[7] B. A. Frasin, Coefficient bounds for certain classes of bi-univalent functions, Hacettepe Journal of Mathematics and Statistics, 43 (3) (2014), 383-389.

[8] B. A. Frasin, M. K. Aouf, New subclasses of bi-univalent functions, Applied Mathematics Letters, 24 (2011), 1569-1573.

[9] Q. Khan, M. Arif, M. Raza, G. Srivastava, H. Tang, Some applications of a new integral operator in q-analog for multivalent functions, Mathematics, 7 (2019), Article ID: 1178,13 pages.

[10] B. Khan, H. M. Srivastava, M. Tahir, M. Darus, Q. Z. Ahmad, N. Khan, Applications of a certain q-integral operator to the subclasses of analytic and bi-univalent functions, AIMS Mathematics, 6 (2021), 1024-1039.

[11] N. Magesh, S. Bulut, Chebyshev polynomial coefficient estimates for a class of analytic bi-univalent functions related to pseudo-starlike functions, Afrika Matematika, 29 (1-2) (2018), 203-209.

[12] S. Mahmood, N. Raza, E. S. A. Abujarad, G. Srivastava, H. M. Srivastava, S. N. Malik, Geometric properties of certain classes of analytic functions associated with a q-integral operator, Symmetry 11 (2019), Article ID: 719, 14 pages.

[13] G. Murugusundaramoorthy, C. Selvaraj, O. S. Babu, Coefficient estimates for Pascutype subclasses of bi-univalent functions based on subordination, International Journal of Nonliner Science, 19 (1) (2015), 47-52. 
[14] S. Ruscheweyh, New criteria for univalent functions, Proceedings American Mathematical Society, 49 (1975), 109-115.

[15] B. Şeker, On a new subclass of bi-univalent functions defined by using Salagean operator, Turkish Journal of Mathematics, 42 (2018), 2891-2896.

[16] L. Shi, Q. Khan, G. Srivastava, J.-L. Liu, M. Arif, A study of multivalent q-starlike functions connected with circular domain, Mathematics, 7 (2019), Article ID: 670, 12 pages.

[17] H. M. Srivastava, Operators of basic (or q-) calculus and fractional q-calculus and their applications in geometric function theory of complex analysis, Iranian Journal of Science and Technology, Transactions A: Science, 44 (2020), 327-344.

[18] H. M. Srivastava, Ş. Altınkaya, S. Yalçin, Certain subclasses of bi-univalent functions associated with the Horadam polynomials, Iranian Journal of Science and Technology, Transactions A: Science, 43 (2019), 1873-1879.

[19] H. M. Srivastava, S. S. Eker, S. G. Hamidi, J. M. Jahangiri, Faber polynomial coefficient estimates for bi-univalent functions defined by the Tremblay fractional derivative operator, Bulletin of the Iranian Mathematical Society, 44 (1) (2018), 149-157.

[20] H. M. Srivastava, S. Gaboury, F. Ghanim, Initial coefficient estimates for some subclasses of m-fold symmetric bi-univalent functions, Acta Mathematica Scientia Series B English Edition, 36 (2016), 863-871.

[21] H. M. Srivastava, S. Gaboury, F. Ghanim, Coefficient estimates for some general subclasses of analytic and bi-univalent functions, Afrika Matematika, 28 (2017), 693-706.

[22] H. M. Srivastava, A. K. Mishra, P. Gochhayat, Certain subclasses of analytic and bi-univalent functions, Applied Mathematics Letters, 23 (2010), 1188-1192.

[23] H. M. Srivastava, A. Motamednezhad, E. A. Adegani, Faber polynomial coefficient estimates for bi-univalent functions defined by using differential subordination and a certain fractional derivative operator, Mathematics, 8 (2020), Article ID: 172, 12 pages.

[24] H. M. Srivastava, N. Raza, E. S. A. AbuJarad, G. Srivastava, M. H. AbuJarad, Fekete-Szegö inequality for classes of $(p, q)$-starlike and $(p, q)$-convex functions, Revista de la Real Academia de Ciencias Exactas, Físicas y Naturales. Serie A. Matemáticas (RACSAM), 113 (2019), 3563-3584.

[25] H. M. Srivastava, A. K. Wanas, Initial Maclaurin coefficient bounds for new subclasses of analytic and $m$-fold symmetric bi-univalent functions defined by a linear combination, Kyungpook Mathematical Journal, 59 (3) (2019), 493-503.

[26] H. M. Srivastava, A. Zireh, S. Hajiparvaneh, Coefficient estimates for some subclasses of $m$-fold symmetric bi-univalent functions, Filomat, 32 (2018), 3143-3153.

[27] H. Tang, H. M. Srivastava, S. Sivasubramanian, P. Gurusamy, The Fekete-Szegö functional problems for some subclasses of $m$-fold symmetric bi-univalent functions, Journal of Mathematical Inequalities, 10 (2016), 1063-1092.

[28] A. K. Wanas, Bounds for initial Maclaurin coefficients for a new subclasses of analytic and $m$-fold symmetric bi-univalent functions, TWMS Journal of Applied and Engineering Mathematics, 10 (2) (2020), 305-311. 
[29] A. K. Wanas, Applications of Chebyshev polynomials on $\lambda$-pseudo bi-starlike and $\lambda$-pseudo bi-convex functions with respect to symmetrical points, TWMS Journal of Applied and Engineering Mathematics, 10 (3) (2020), 568-573.

[30] A. K. Wanas, A. L. Alina, Applications of Horadam polynomials on Bazilevič biunivalent function satisfying subordinate conditions, Journal of Physics: Conference Series, 1294 (2019), 1-6.

[31] A. K. Wanas, A. H. Majeed, On subclasses of analytic and $m$-fold symmetric bi-univalent functions, Iranian Journal of Mathematical Sciences and Informatics, 15 (2) (2020), 51-60.

[32] A. K. Wanas, A. H. Majeed, Chebyshev polynomial bounded for analytic and biunivalent functions with respect to symmetric conjugate points, Applied Mathematics E-Notes, 19 (2019), 14-21.

[33] A. K. Wanas, H. Tang, Initial Coefficient estimates for a classes of m-fold symmetric bi-univalent functions involving Mittag-Leffler function, Mathematica Moravica, 24 (2) (2020), 51-61.

\author{
Serap Bulut \\ Faculty of Aviation and Space Sciences \\ Kocaeli University \\ Arslanbey Campus \\ 41285 KaRtepe/Kocaeli \\ TURKEY \\ E-mail address: serap.bulut@kocaeli.edu.tr
}

